\title{
In vitro evaluation of the response of human tendon-derived stromal cells to a novel electrospun suture for tendon repair
}

\author{
Andrey Nezhentsev $^{1}$ | Roxanna E. Abhari ${ }^{1}$ (D) | Mathew J. Baldwin ${ }^{1}$ | Jolet Y. Mimpen ${ }^{1}$ | \\ Edyta Augustyniak $^{1}$ | Mark Isaacs $^{2,3}$ | Pierre-Alexis Mouthuy ${ }^{1}$ | Andrew J. Carr ${ }^{1}$ | \\ Sarah J. B. Snelling ${ }^{1}$
}

\author{
${ }^{1}$ Nuffield Department of Orthopaedics, \\ Rheumatology, and Musculoskeletal \\ Sciences, University of Oxford, Oxford, UK \\ ${ }^{2}$ Department of Chemistry, University \\ College London, London, UK \\ ${ }^{3}$ HarwellXPS, Research Complex at \\ Harwell, Rutherford Appleton Laboratories, \\ Harwell Campus, UK

\section{Correspondence} \\ Roxanna E. Abhari, NDORMS, University \\ of Oxford, Botnar Research Centre, Old \\ Road, Headington, Oxford OX37LD, UK. \\ Email: roxanna.abhari@medschool.ox.ac.uk \\ Funding information \\ NIHR Oxford Biomedical Research Centre
}

Recurrent tears after surgical tendon repair remain common. Repair failures can be partly attributed to the use of sutures not designed for the tendon cellular niche nor designed for the promotion of repair processes. Synthetic electrospun materials can mechanically support the tendon while providing topographical cues that regulate cell behavior. Here, a novel electrospun suture made from twisted polydioxanone (PDO) polymer filaments is compared to PDS II, a clinically used PDO suture currently utilized in tendon repair. We evaluated the ability of these sutures to support the attachment and proliferation of human tendon-derived stromal cells using PrestoBlue and scanning electron microscopy. Suture surface chemistry was analyzed using x-ray photoelectron spectroscopy. Bulk RNA-Seq interrogated the transcriptional response of primary tendon-derived stromal cells to sutures after 14 days. Electrospun suture showed increased initial cell attachment and a stronger transcriptional response compared with PDS II, with relative enrichment of pathways including mTorc1 signaling and depletion of epithelial-to-mesenchymal transition. Neither suture induced transcriptional upregulation of inflammatory pathways compared to baseline. Twisted electrospun sutures therefore show promise in improving outcomes in surgical tendon repair by allowing increased cell attachment while maintaining an appropriate tissue response.

\section{K E Y W O R D S}

electrospinning, electrospun suture, PDS II, polydioxanone, tendon repair, tissue engineering

\section{1 | INTRODUCTION}

Tendon injuries are common and cause pain and reduced quality of life for patients. Rotator cuff tendon tears alone affect around $50 \%$ of those over 66 years of age, ${ }^{1}$ and many of these patients require surgical repair. ${ }^{2}$ However, despite exploration of different suture methods, surgical tendon repairs have poor outcomes due to suture pull-through or tissue re-tears, with

Andrey Nezhentsev and Roxanna E. Abhari joint first authors.
$40 \%$ of rotator cuff repairs failing within one year. ${ }^{3}$ This failure to successfully repair torn tendons causes long-term disability and represents a significant socioeconomic cost. ${ }^{4}$

Suture-based re-joining of tendon ends is the current gold standard surgical treatment for tendon injuries, but currently used sutures have been repurposed from other anatomical sites and have not been designed for the tendon niche. Clinically used sutures are manufactured from synthetic polymers with high tensile strength to provide mechanical augmentation. However, their topographical

This is an open access article under the terms of the Creative Commons Attribution License, which permits use, distribution and reproduction in any medium, provided the original 
characteristics (including fiber diameter) are dissimilar to native tendon, which is composed of multiple aligned small diameter collagen fibers. Furthermore, cells do not integrate with the material, ${ }^{5}$ demonstrated by acellular zones forming around sutured sites. ${ }^{6,7}$ This lack of infiltration and integration likely contributes to poor tendon healing at the suture-tissue interface. In addition, immune and stromal cells drive a foreign body response to implantable materials including sutures. This can result in chronic inflammation, which can hamper endogenous repair and lead to tissue failure. ${ }^{8}$

The use of sutures that enable cellular attachment and proliferation, and that do not raise a chronic inflammatory response may improve the efficacy of tendon repair. ${ }^{5}$ Electrospun materials show particular promise and can be produced from synthetic and clinically approved materials, including polydioxanone (PDO), which is an absorbable polymer used to produce PDS II sutures currently deployed in tendon repair. The high surface area and porosity of electrospun materials together with the ability to manufacture them with fiber diameters similar to the collagen fibers in native tendons provide biomimicry not afforded by conventional sutures. ${ }^{9}$ Electrospun materials also promote infiltration and proliferation of stromal cells, including those derived from tendon, and induce expression of tenogenic markers. ${ }^{10-12}$ Surface chemistry, fiber diameter, and alignment of materials can tune cell behavior, and this can be exploited to drive repair. However, when manipulating the mechanical and topographical properties of a clinically approved material it is important to define the cellular response to its specific material properties.

Twisting and braiding electrospun strands into bundles can direct cells to attach and grow in a parallel network, similar to the macroscopic architecture of tendon. ${ }^{14,15}$ The morphology and proliferation of tendon-derived stromal cells cultured on electrospun materials have been previously described, but only a small number of selected genes were assessed in tendon-derived stromal cell gene expression analyses ${ }^{14,16-18}$ and response. ${ }^{7,13}$ Previous work has also described the twisting of electrospun PDO fibers into prototype multifilament yarns that have similar a tensile strength to currently used sutures and that resemble the hierarchical structure of tendons. ${ }^{13}$ Following in vivo tendon injury in a rat and sheep model, prototype electrospun sutures supported cellular infiltration with a minimal inflammatory response. Understanding how tendon cells interact with the material in vitro can therefore help predict how the electrospun suture will interact and integrate with tendon in vivo. The effect of twisted electrospun sutures on the global transcriptional profile of tendon-derived stromal cells in vitro or in vivo is not well explored. It therefore remains necessary to understand the response of tendon cells to sutures developed for their surgical repair, compared with sutures currently used in surgery.
The overarching aim of this in vitro study was to assess the potential of twisted electrospun PDO sutures in tendon repair. This builds on our previously published work ${ }^{13,19}$ and uses a modified electrospun suture that ensures uniformity of fiber diameter, and a high tensile strength and hierarchical structure that have potential to both mechanically and biologically support repair. We hypothesized that a twisted electrospun suture would promote tendon-derived stromal cell attachment and proliferation and induce a pro-reparative gene expression profile.

\section{MATERIALS AND METHODS}

\subsection{Manufacture of electrospun sutures}

Electrospun monofilaments of PDO were produced according to previously described methods. ${ }^{40}$ Polydioxanone (PDO; Riverpoint Medical, Portland, USA) was dissolved in 1,1,1,3,3,3-hexafluoroisopropanol (HFIP; Halocarbon Product Corporation, Atlanta, USA) at a concentration of $7 \%(\mathrm{w} / \mathrm{v})$ in the presence of pyridine. Electrospinning was performed in a glove box using a single-nozzle highvoltage power supply system (30 kV, SL30P30/230; Spellman, West Sussex, UK) and a syringe pump (World Precision Instruments Limited, Florida, USA). Filaments were produced by using a thin stainless steel wire $(100 \mu \mathrm{m}$ in diameter; Goodfellow, Huntingdon, UK) as a collector. The wire was moved underneath the nozzle at a speed of $0.5 \mathrm{~mm} / \mathrm{s}$ with a bespoke winding unit. Electrospun filaments were then continuously wound up onto a motorized filament spool, separating them from the wire collector. They were then stretched manually to $3 \mathrm{x}$ their original length. Then, $5 \times 50 \mathrm{~cm}$ stretched monofilaments were taped at one end parallel to each other, and their other end was taped to a flathead screwdriver. 300 turns/meter were performed in the $\mathrm{S}$ twist direction to create a ply yarn. 7 ply yarns were taped down and taped to a screwdriver as before, and 150 turns/meter were performed in the $\mathrm{Z}$ twist direction to create electrospun sutures. The opposite twist directions make the material "twist-neutral," increasing its stability. The sutures were wound tightly around a spool, taped down, and annealed for $3 \mathrm{hr}$ at $65^{\circ} \mathrm{C}$ in a laboratory oven (Genlab, Widnes, UK). The samples were stored in a desiccator (Bohlender, Grünsfeld, Germany) when not being handled to protect it from humidity.

Four 2-cm pieces of electrospun suture and PDS II (Ethicon Inc) were then melted together at both ends by holding near a $200^{\circ} \mathrm{C}$ hot wire (Proxxon, Axminster, UK). This formed mats that could be transferred between tissue culture wells without disrupting cells (Figure S1). The mats were sterilized by submerging in $70 \%$ ethanol for 2 hours and dried overnight. The sutures were washed twice in PBS and 
soaked for 2 hours in D10 medium (DMEM-F12 (Thermo Fisher Scientific) supplemented with $10 \%$ fetal calf serum (Labtech, Melbourn, UK) and 1\% penicillin-streptomycin (Thermo Fisher Scientific)).

\section{$2.2 \quad$ Tendon-derived stromal cell seeding}

Waste healthy mid-body hamstring tendon tissue was collected from four male patients aged 20-43 ( $\mathrm{SD} \pm 9.4 \mathrm{yr}$ ), BMI 21.46-27.76 (SD $\pm 2.77 \mathrm{~kg} / \mathrm{m} 2)$, during anterior cruciate ligament reconstruction. Tendon-derived stromal cells were extracted and expanded from tendon explants as previously described $^{18}$ (S2). 50,000 tendon-derived stromal cells (passage 3) were seeded dropwise onto the suture mats, or directly into empty wells for tissue culture plastic (TCP) controls ( 3 technical repeats for each patient). TCP controls were used later to determine whether changes in gene expression were caused by cell-instructive cues provided by the suture materials. After 4 hours, mats were transferred into a new 12well plate. The percentage of seeded cells that remained on the electrospun suture and PDSII after transferring to a new well was evaluated (S3).

\section{3 | Scanning electron microscopy}

Suture pieces were fixed for $10 \mathrm{~min}$ in $2.5 \%$ glutaraldehyde (Sigma-Aldrich, St. Louis, Missouri, USA), rinsed twice in deionized water, and dehydrated in a graded series of ethanol concentrations (Sigma-Aldrich) (40\%, 70\%, 90\%, $95 \%, 100 \%$ ), for 2 min each. The sutures were left for $24 \mathrm{~h}$ in $100 \mu \mathrm{L}$ of hexamethyldisilazane (Alfa Aesar, Haverhill, MA, USA). The sutures were gold-coated using the SC7620 Mini Sputter Coater System (Quorum Technologies, Lewes, UK). The EVO LS15 Variable Pressure Scanning Electron Microscope (Carl Zeiss AG, Oberkochen, Germany) was used to capture images. Images were taken at 200X magnification to visualize suture structure and at 2000X magnification to visualize attached cells.

\section{4 | Cell viability}

Measurements of cell viability were performed by incubating cells seeded on sutures in 10\% PrestoBlue for 2 hours on days $1,4,7,11$, and 13 after seeding. PrestoBlue was prepared by mixing 10X PrestoBlue Cell Viability Reagent (Thermo Fisher Scientific) with medium pre-warmed to $37^{\circ} \mathrm{C}$. The sutures were incubated in $2 \mathrm{~mL}$ of $10 \%$ PrestoBlue for $2 \mathrm{~h}$ at $37^{\circ} \mathrm{C}$. An empty well with $10 \%$ PrestoBlue was used to correct for background fluorescence. Due to insufficient numbers of cells, no calibration curve was generated for the PrestoBlue assay. However, previous experiments have shown that the number of tendon-derived stromal cells seeded and cultured on the sutures within 14 days is well within the limit of linearity of this assay's dose-response curve. Changes in fluorescence could therefore be used to indicate changes in cell number.

$100 \mu \mathrm{L}$ samples $(\mathrm{n}=3)$ were taken from each of the wells and pipetted into a 96-well plate (Greiner Bio-One). Bubbles in the well were popped using a sterile needle (BD Microlance; Franklin Lakes, NJ, USA). Fluorescence was measured using a FLUOstar Omega Microplate Reader (BMG Labtech, Aylesbury, UK) at $544 \mathrm{~nm}$ excitation and $590 \mathrm{~nm}$ emission. For each experiment, the gain was adjusted to a well with solution from cells cultured on electrospun suture, where the highest fluorescence was expected. Resazurin in the PrestoBlue Cell Viability Reagent (Thermo Fisher Scientific) is reduced by living cells to the fluorescent resorufin, so the fluorescence indicated cell viability. A mean fluorescence was calculated from the samples taken from each well $(n=3)$. The sutures were moved into fresh medium pre-warmed to $37^{\circ} \mathrm{C}$ after each assay.

\subsection{RNA extraction}

RNA was extracted from hamstring tendon-derived stromal cells ( $\mathrm{n}=4$ patients) after 14 days' culture on sutures and TCP after 14 days' culture and at baseline (time of seeding on sutures and TCP). ${ }^{20}$ The sutures were incubated in 700 $\mu \mathrm{L}$ of TRIzol (Thermo Fisher Scientific) to lyse the cells. RNA was extracted using a Direct-zol RNA MicroPrep Kit (Zymo Research, Irvine, CA, USA) according to the manufacturer's protocol. RNA concentration was measured using a NanoDrop (Implen; Westlake Village, CA, USA). Samples were frozen at $-80^{\circ} \mathrm{C}$ until RNA-Seq.

\section{6 $\quad$ RNA sequencing}

Bulk RNA-Seq interrogated the tendon-derived stromal cells' transcriptomic response to the sutures. Libraries were created using a NEBNext Ultra II Directional RNA Library Prep Kit for Illumina (New England Biolabs, Ipswich, MA, USA), and sequencing was performed on an Illumina NextSeq 500 using a NextSeq High Output Kit. A quality control (QC) report was collated using the MultiQC tool (v1.7). ${ }^{21}$ Due to low percentage alignment with the reference genome, one PDS II sample did not pass QC and was excluded from analysis. Principal component analysis plots extracted the components responsible for most of the dataset variance (S4). The DESeq2 package ${ }^{22}$ was utilized to undertake pairwise comparisons of gene expression at 14 days of culture. Geneset enrichment analyses were performed using the cluster 
F I G URE 1 Effect of suture material on cell attachment and viability after 13 days of healthy tendon-derived stromal cell culture. (A-H) SEM images of suture materials at different points during cell culture with healthy human tendon-derived stromal cells (magnification 2000X). Red arrows point to attached cells. (A) Electrospun suture pre-seeding (x200 inset) and at days 1 (C), 7 (E), and 13 (G) after seeding. (B) PDS II pre-seeding and at days 1 (D) (x200 inset), 7 (F), and 13 (H) after seeding. (I) Fluorescence intensity, indicating the number of viable cells in the sample, plotted over a period of 13 days. There was a significantly higher fluorescence intensity from the electrospun suture compared with PDS II at each time point. (J) Fluorescence was also plotted relative to day 1 to indicate proliferation independent of initial cell attachment. No statistically significant differences were seen between electrospun suture and PDS II. (I-J) represent the average results of $n=4$ patient samples cultured on $\mathrm{n}=3$ intra-experimental replicates of each suture. Error bars indicate standard deviation

Profiler package, ${ }^{23}$ and data were visualized using Enhanced Volcano and ggplot2.

\section{7 | X-ray photoelectron spectroscopy}

To assess the surface chemistry of sutures, sutures were flattened into sheets by a hydraulic press (Specac, Orpington, UK) set to 8 tonnes for 30 seconds, and mounted onto a glass slide using double-sided carbon tape. $\mathrm{x}$-ray photoelectron spectroscopy (XPS) measurements were made using an AXIS Supra (Kratos Analytical, Stretford, UK) (S6).

\section{8 | Data analysis}

GraphPad Prism 8 (GraphPad Software Inc, San Diego, CA, USA) was used for statistical analysis of XPS and cell viability data. The D'Agostino-Pearson test was used to test the normality of the biological replicates. Unpaired t tests were used to determine whether there was a difference in between the two suture samples. The Holm-Sidak method was used to correct for multiple comparisons. Results were deemed statistically significant when $P<.05$, and statistical significance is displayed in shorthand as $* P<.05, * * P<.01, * * * P<.001$, and $* * * * P<.0001$. $\mathrm{R}$ and $\mathrm{R}$ packages (S5) were used to analyze gene expression data.

\section{3 | RESULTS}

\section{1 | Initial cell attachment was higher on twisted electrospun suture than PDS II}

To compare the ability of electrospun and PDS II sutures to support attachment and proliferation of tendon-derived stromal cells, cells were cultured for 13 days on each surface and assessed using SEM imaging and PrestoBlue viability assays. SEM images showed cells attaching to and proliferating on both sutures, with cell coverage increasing throughout the duration of the experiment (Figure 1A-H). PrestoBlue viability analysis was used to give a surrogate measure of relative cell number over 13 days of tissue culture. The number of viable cells was higher on the electrospun suture compared with PDS II on day $1(P<.001)$ (Figure 1I), indicating higher initial stromal cell attachment. The number of cells present on electrospun sutures remained higher throughout the culture period, with the electrospun suture containing 4 times as many cells than PDS II by day $13(P<.0001)$ (Figure $1 \mathrm{I})$. When cell number was baseline-corrected relative to day 1 , there were no differences in proliferation rates on each suture material (Figure 1J).

\section{2 | Electrospun suture elicits a stronger transcriptional response in tendon-derived stromal cells than PDS II}

The transcriptome of tendon-derived stromal cells at baseline and cultured for 14 days on the sutures or TCP was evaluated using bulk RNA-Seq. Differentially expressed genes (Padj < 0.05, LFC \pm 1$)$ of cells cultured for 13 days on electrospun sutures, PDS II, and TCP compared with baseline are shown using volcano plots (Figure 2). After 14 days' culture, 1849, 667, and 61 genes were differentially expressed on electrospun sutures, PDS II, and TCP, respectively, when compared to baseline (Figure 2A-C). When directly comparing the transcriptome of cells cultured on electrospun and PDS II sutures on day 13, 122 genes were differentially expressed (Figure 2D).

Hallmark gene-set enrichment analysis was used to analyze whether the differentially expressed genes significantly contributed to changes in 50 well-defined biological processes (Figure 3). While both sutures upregulated expression of gene sets associated with cell cycle progression (MYC and E2F targets) and DNA repair, the electrospun suture leads to more pronounced changes than PDS II. Additional differences between the two sutures could be seen in pathways related to inflammation (IL6-JAK-STAT3 signaling and TNF- $\alpha$ signaling via NF- $\kappa B$ ) and hypoxia, which were significantly downregulated by PDS II, which is similar to TCP but not the electrospun suture. When directly comparing the two sutures, 18 gene sets were differentially regulated (Figure 3B) including enrichment for Mtorc 1 signaling and Myc targets, and a relative reduction in the gene sets belonging to the epithelial-to-mesenchymal transition pathway in cells cultured on electrospun suture compared with PDS II sutures. Gene ontology gene-set enrichment analysis found that 


\section{Days after} seeding

0

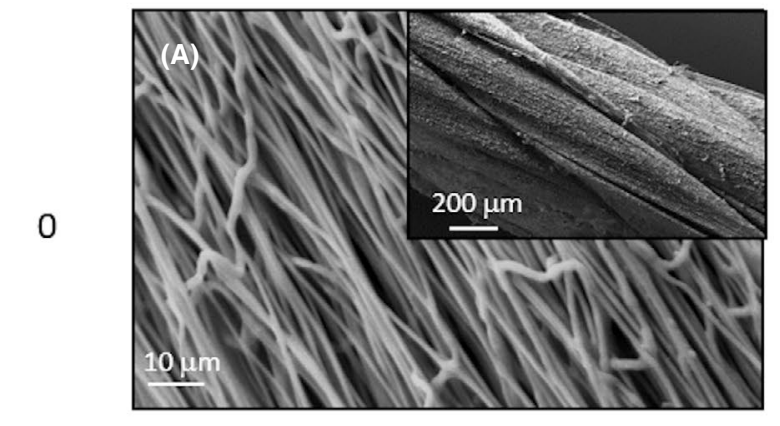

Electrospun suture

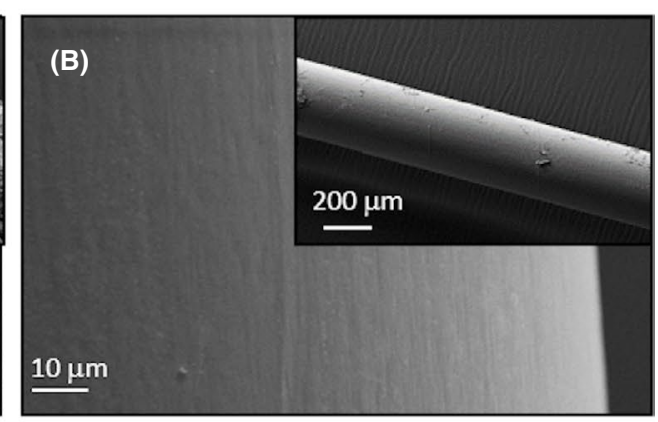

1
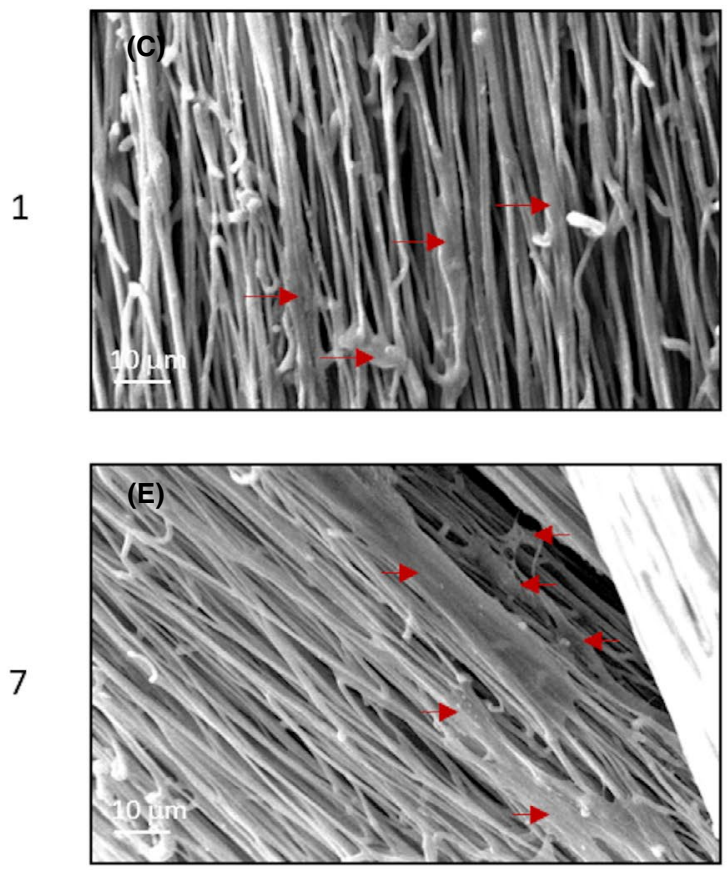

13

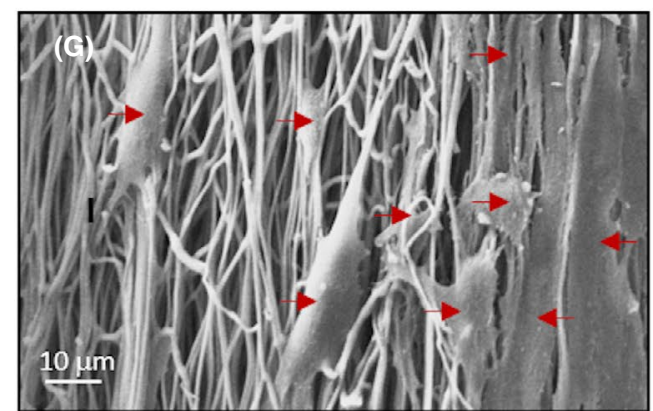

(I)
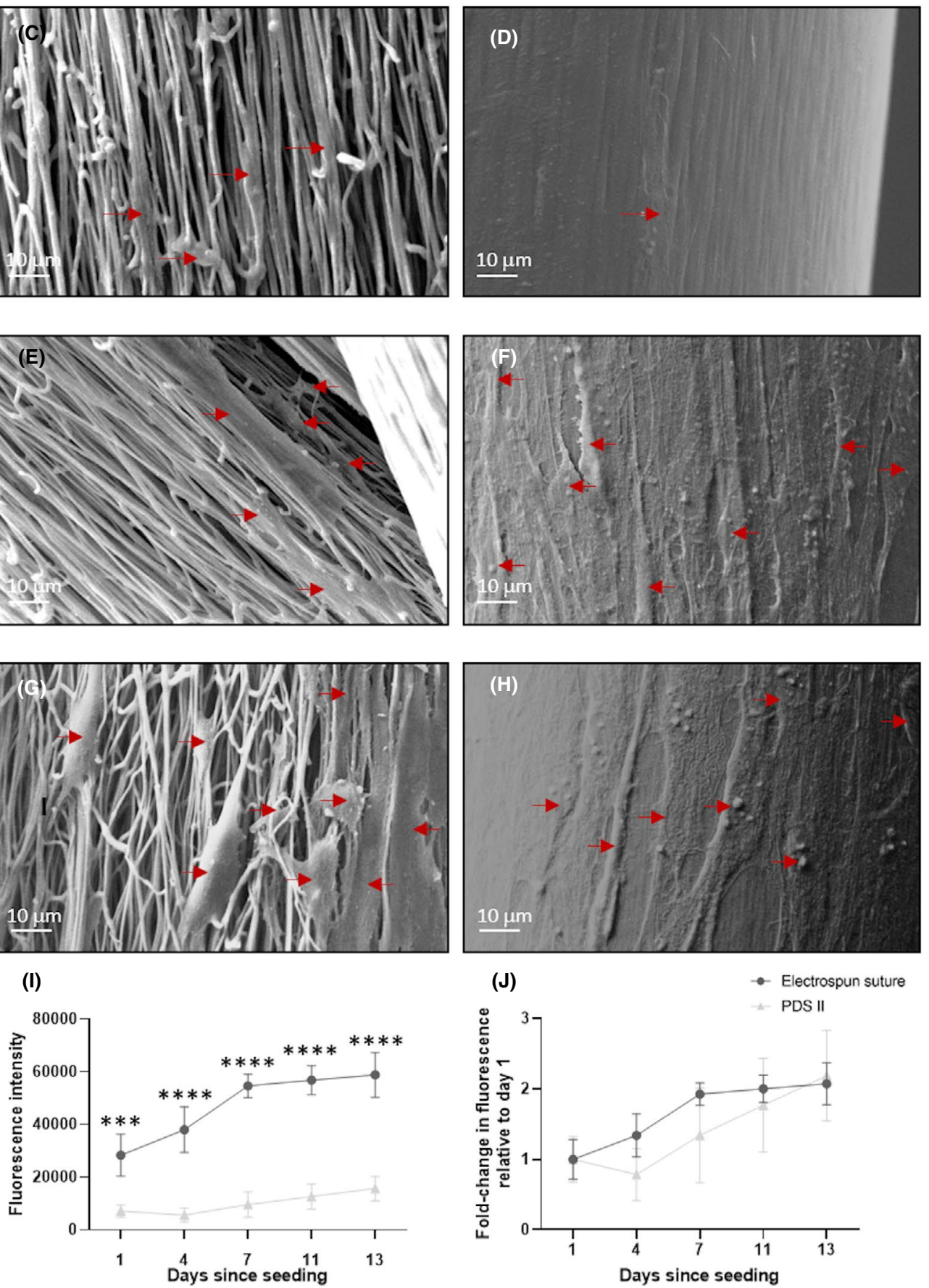


\section{(A) ES vs T0}

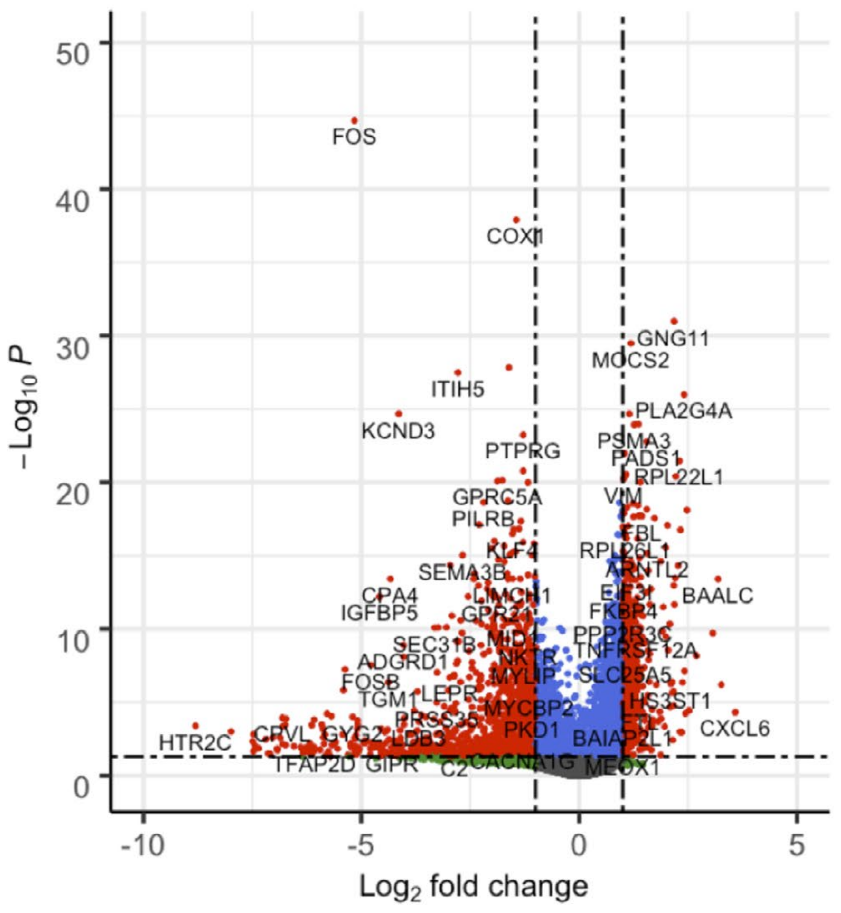

- NS - Log2 fold-change - P value - P value \& Log2 fold-change

(C)

\section{TCP vs To}

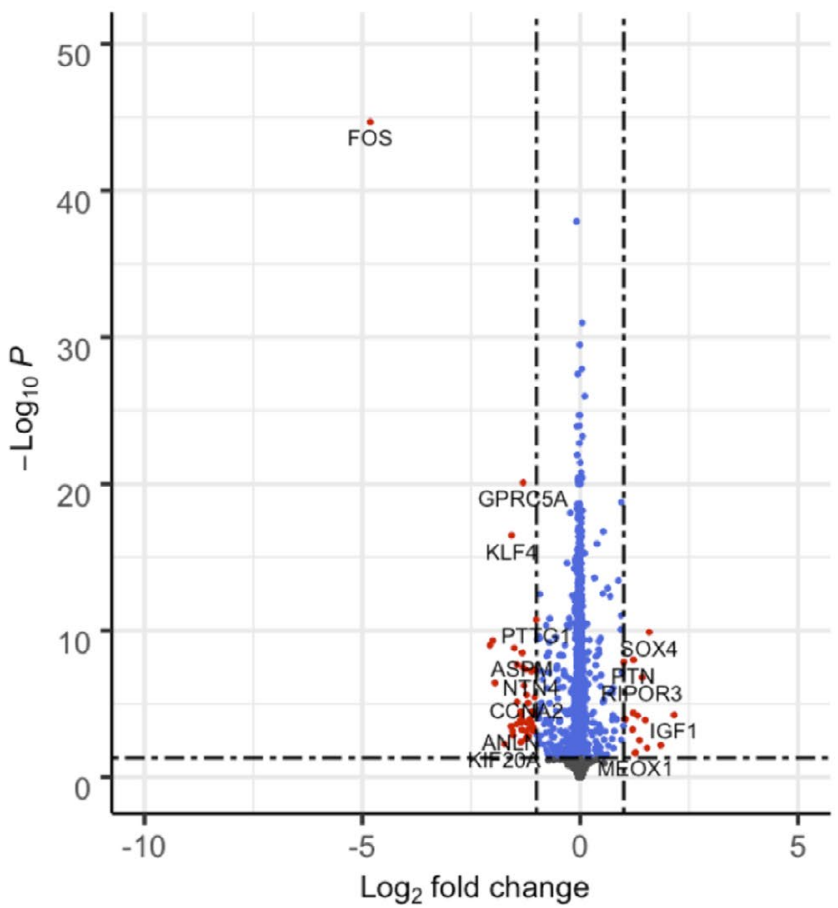

\section{(B) PDS vs T0}

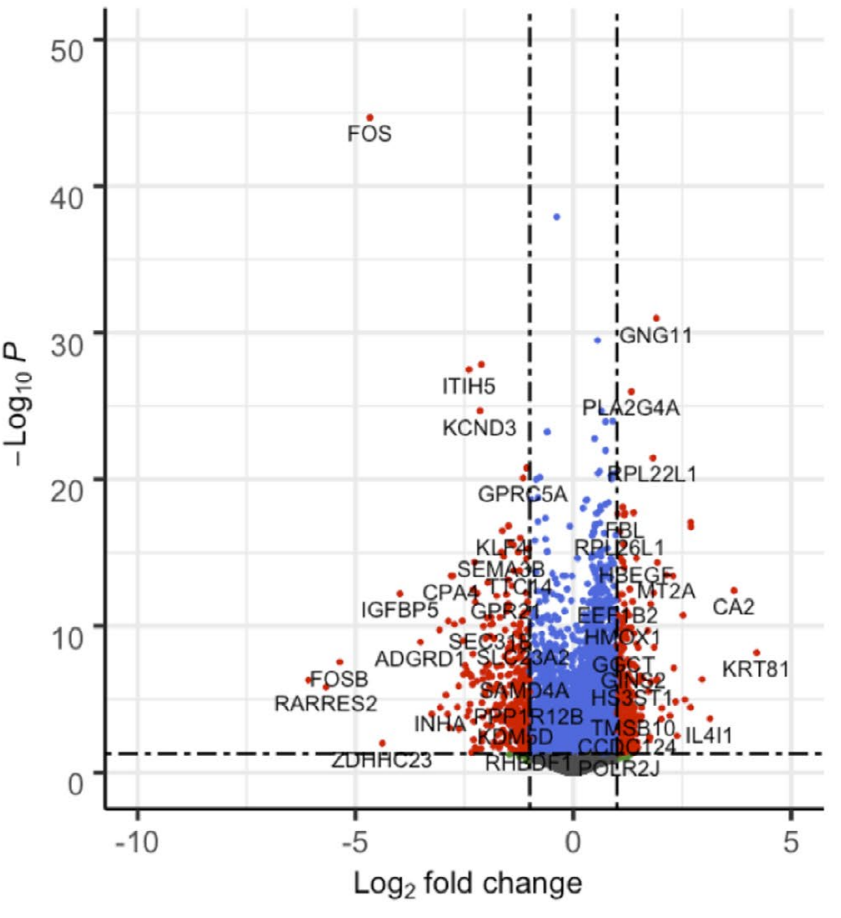

- NS - Log2 fold-change - P value - P value \& Log2 fold-change

(D)

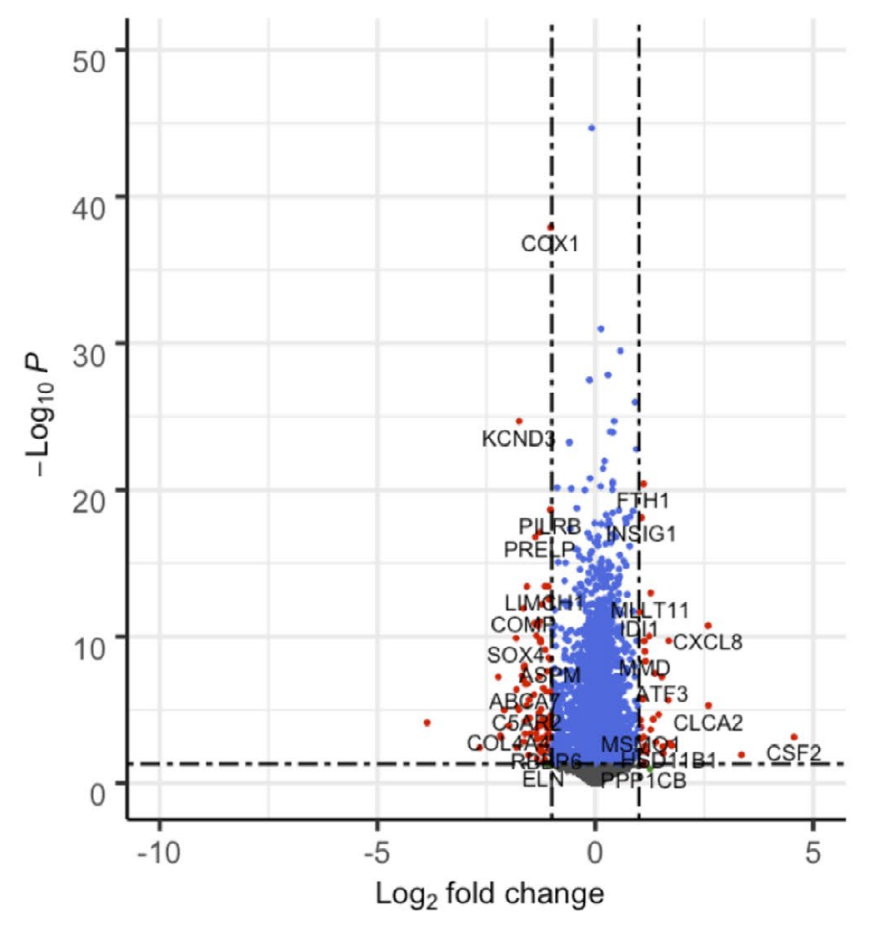

F I G U R E 2 Differentially expressed genes of tendon-derived stromal cells cultured on (A) electrospun (ES) sutures, (B) PDS II sutures, or (C) tissue culture plastic control for 14 days compared with baseline control (T0). (D) Differentially expressed genes of cells cultured on either ES or PDS II for 14 days. Genes meeting the statistical significance $(P<.05)$ and have a $\log 2$ fold change of at least \pm 1 are shown in red, $\mathrm{n}=4$ patients 


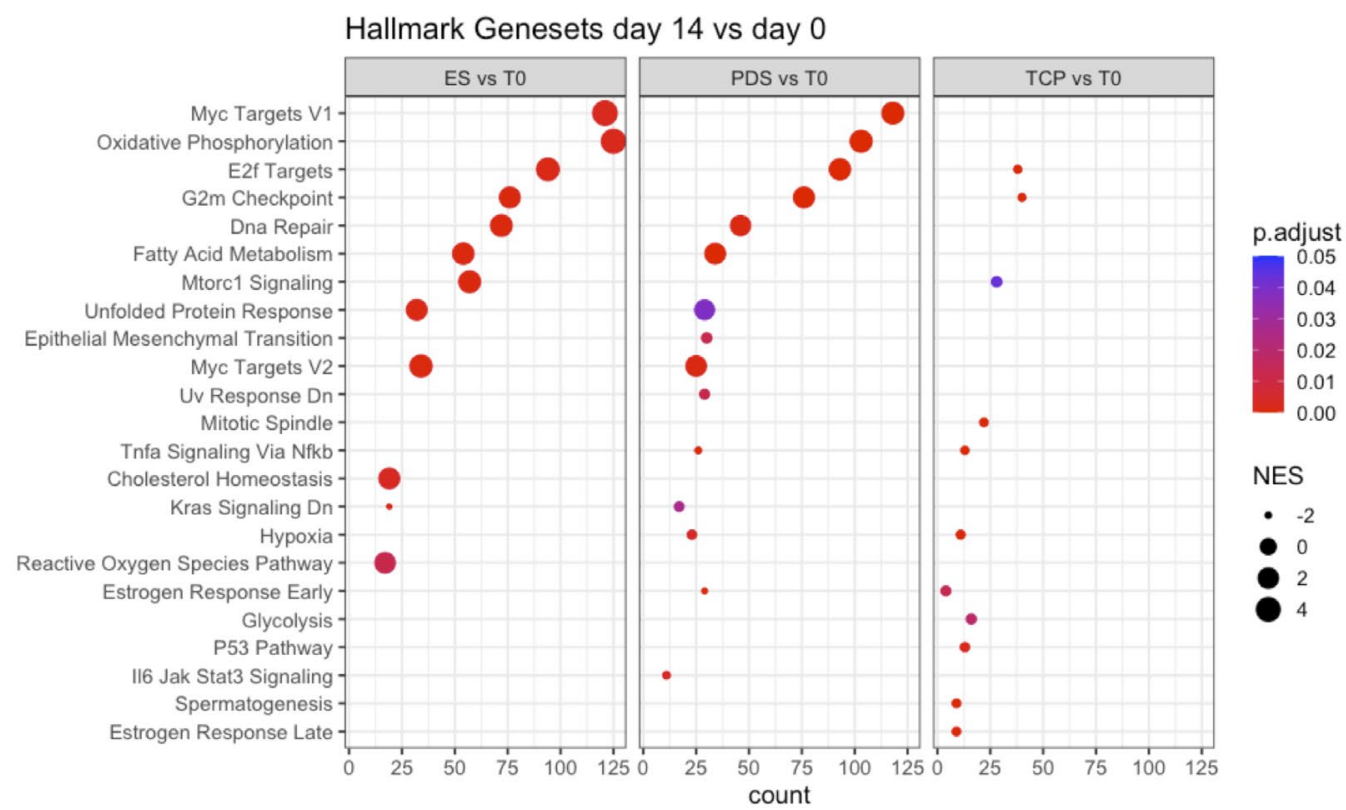

Hallmark Genesets ES vs PDS

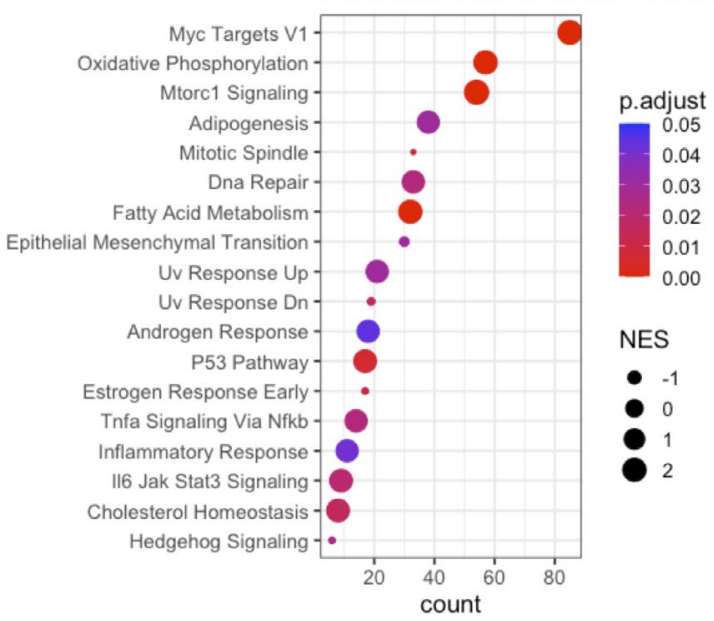

GO Genesets ES vs PDS

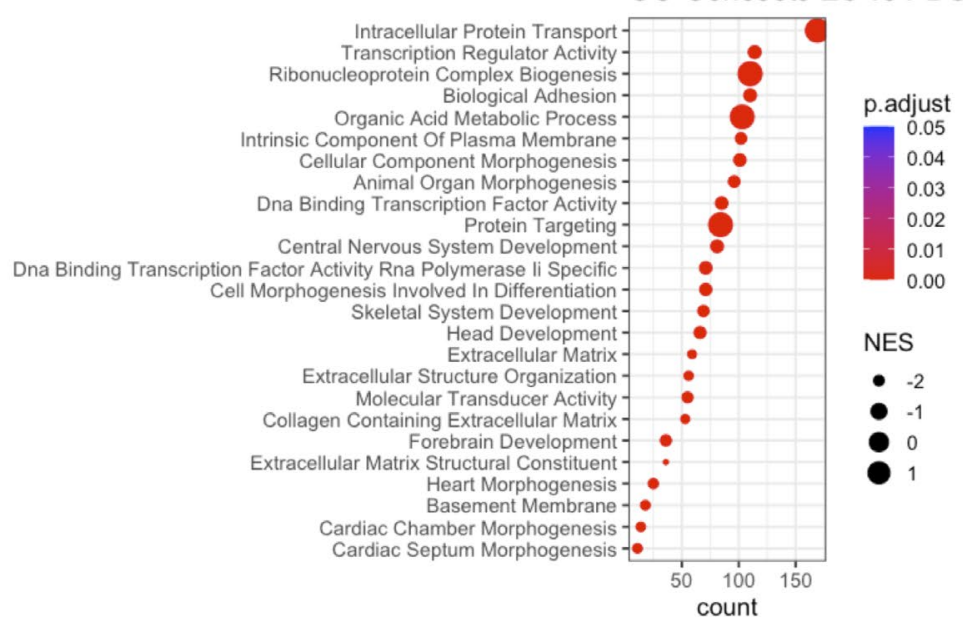

F I G U R E 3 Changes in hallmark or Gene Ontology (GO) gene sets based on differentially expressed genes. Differentially expressed genes of tendon-derived stromal cells cultured on electrospun (ES) sutures, PDS II sutures, or tissue culture plastic (TCP) control significantly contributed to changes in well-defined biological processes. Enrichment analysis of gene clusters after 14 days of cell culture on the materials was compared to baseline control (T0), and gene clusters after culture on ES and PDS II were compared directly. NES = normalized enrichment score

differentially regulated genes between electrospun and PDS II sutures contributed to changes in biological adhesion, cellular component morphogenesis, and (collagen-containing) extracellular matrix.

\subsection{Local surface chemistry of electrospun sutures and PDS II is similar}

While electrospun and PDS II sutures are both made of PDO, these results show that tendon-derived stromal cells cultured on these sutures have significant differences in attachment and transcriptional response. Differences in chemical functional groups at the suture surface may mediate altered serum protein attachment, leading to the observed differences in tendon-derived stromal cell response electrospun and PDS II sutures. ${ }^{24}$ To establish whether differences in the structure or manufacturing processes of the sutures had resulted in differences in surface chemistry, XPS analysis was used to determine the functional groups present on the surface of the suture. Both sutures are made from PDO, containing C-$\mathrm{C} / \mathrm{C}-\mathrm{H}, \mathrm{C}-\mathrm{O}$, and $\mathrm{C}=\mathrm{Oy}$ functional groups, and these groups were all present on the surface of both sutures. Although there were subtle alterations in the abundance of $\mathrm{C}-\mathrm{O}$ groups on the surface of electrospun compared with PDS II sutures, this did not reach statistical significance (Figure 4), suggesting that differences in structure and manufacturing processes do not strongly affect suture surface chemistry. 


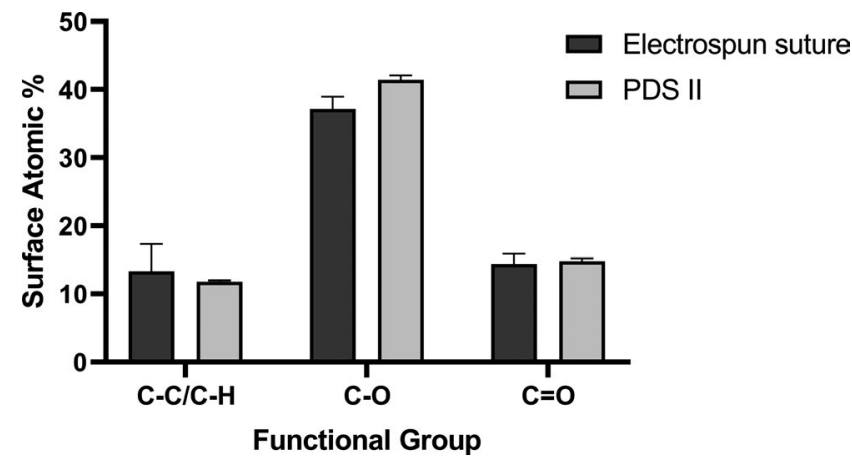

F I G URE 4 Suture surface characterization using x-ray photoelectron spectroscopy. High-resolution carbon and oxygen XPS spectra, comparing carbon in various chemical states at the surface of the sutures (surface atomic \%). No statistically significant differences in suture surface chemistry were observed. The figure represents the average results of $n=3$ points on a suture sample. Graphs show mean $+\mathrm{SD}$

\section{4 | DISCUSSION}

Tendon disease is common, and surgical repair of torn tendons is prone to failure. Electrospun materials that mimic the hierarchical structure of tendon tissues could be used to support endogenous tissue repair. This work aimed to investigate the potential of a twisted electrospun suture in the surgical repair of tendon tears. Tendon-derived stromal cells showed increased attachment to electrospun sutures. We also demonstrated that electrospun sutures induced a distinct and stronger tendon-derived stromal cell transcriptomic response when compared to PDS II sutures.

Tendon-derived stromal cells attached to and proliferated on both electrospun sutures and PDS II, but initial cell attachment to electrospun sutures was significantly higher. There were no statistically significant differences in the sutures' surface chemistry, which would have meant similar serum protein attachment and subsequent cell attachment. However, it is likely that the greater cell attachment to electrospun suture was caused by its highly textured surface and high surface area, compared with the smoother surface of PDS II. Indeed, electrospun sutures are composed of multiple twisted fibers with diameters that not only resemble collagen fibrils but have also been shown to promote fibroblast adhesion and infiltration. ${ }^{25,26}$ The attachment of greater cell numbers could potentially lead to relative increases in ECM production, possibly accelerating tendon repair. Electrospun sutures have previously been shown to promote cellular infiltration in vivo and improved tissue integration, which could reduce the rate of suture pull-through. $^{7}$

To investigate the sutures' effects on gene expression, RNA-Seq was performed on healthy tendon-derived stromal cells after 14 days' culture on TCP, electrospun, and PDS II sutures. Ideally, sutures should stimulate a gene expression profile indicative of wound healing, upregulating pathways associated with cell proliferation and repair, without uncontrolled or sustained upregulation of fibrotic or inflammatory pathways. ${ }^{8,27}$ Tendon-derived stromal cells cultured on electrospun suture and PDS II both upregulated gene sets associated with the cell cycle, indicating enhanced cellular proliferation, and upregulated mTORC1 signaling, indicating upregulation of pathways relating to wound healing, protein synthesis, and tendon maturation. ${ }^{28}$ These results were more pronounced for electrospun sutures. PDS II is regarded as an immune-compatible suture, based on favorable cellular inflammatory responses in rodent models of soft tissue repair, and therefore, the similarities in response to PDS II and electrospun sutures support exploration of electrospun sutures for tendon repair. ${ }^{29-31}$ Tendon-derived stromal cells cultured on electrospun sutures also downregulated epithelial-to-mesenchymal transition and extracellular matrix gene sets, and upregulated NF-kB gene sets when compared to PDS II. This suggests they induce a wound-healing response that is not strongly fibrotic, potentially minimizing formation of weak scar tissue, which may lead to tendon repair failure. ${ }^{32}$ Surface chemistry, porosity, and topography are all able to regulate fibroblast behavior and may have contributed to the differences in transcriptional profile of tendon-derived stromal cells cultured on PDS II and electrospun sutures. Few genes were differentially expressed after 14 days of culture on TCP, indicating that PDS II and electrospun sutures provided cell-instructive cues and that gene expression changes in these sutures were not due to temporal changes due to prolonged culture alone. Although PDS II is considered biocompatible, it was not designed for repairing damaged tendon tissue. By allowing increased tendon-derived stromal cell attachment while not inducing a fibrotic transcriptional response, electrospun sutures could therefore improve the outcomes of surgical tendon repair when compared to currently used sutures.

\section{5 | PERSPECTIVES}

\section{1 | Future directions and limitations}

This work has a number of limitations. The electrospun suture is more porous and has a larger diameter than the PDSII suture, which could have influenced initial cell attachment. However, the electrospun suture is meant to be used in its current form (with a larger diameter), so comparing to PDSII is useful to evaluate whether the electrospun suture could provide value over currently used sutures. Tendon-derived stromal cells from diseased tendons would better recapitulate the response of pathological tissue to the materials. Tissue from massive rotator cuff tears $(>5 \mathrm{~cm})$ 
is in greatest need of improved augmentation materials, as they have the highest rate of failure. ${ }^{33}$ Finally, during surgical repair, the damaged tendon is rapidly infiltrated by macrophages ${ }^{34}$ which could alter the cellular environment that biomaterials are exposed to. ${ }^{35,36}$ Tendon-derived stromal cells co-cultured with monocyte-derived macrophages would more accurately recapitulate the diseased tendon niche.

As bulk RNA-Seq can mask the response of cell subsets, it is also necessary to explore cell type-specific responses to various sutures. Recent reports have used single-cell RNASeq of healthy and diseased tendon to identify 8 or more subpopulations of tendon cells, including 5 distinct types of tendon-derived stromal cells. ${ }^{37,38}$

Endogenous tendon repair lasts longer than the 14 days of tissue culture conducted in this study and occurs within a loading environment. ${ }^{39}$ However, it should be noted that Rashid et al examined the in vivo response of English Mule sheep tendon 3 months after surgical repair with a similar electrospun suture to the one described in this paper. ${ }^{7}$ There were little inflammation and extensive cellular infiltration into the suture upon histological examination.

\section{6 | CONCLUSIONS}

This study compared the tendon-derived stromal cell response to clinically used PDS II and a novel twisted PDO electrospun suture. Compared to PDS II, a currently used and safe suture, electrospun sutures demonstrated greater cell attachment and tendon-derived stromal cell transcriptomic response indicative of cell proliferation and wound healing without significant fibrosis. These results indicate that electrospun suture is a promising material that may improve the outcomes of surgical tendon repair.

\section{ACKNOWLEDGMENTS}

We would like to thank the NIHR Oxford Biomedical Research Centre for funding this work. We also thank the Oxford Musculoskeletal Biobank, Louise Appleton, Debra Beazley, Kim Wheway, and Bridget Watkins for the collection of human hamstring tendons. We would also like to thank Hayley Morris, Joana Martins, and Antonina Lach for their guidance and assistance in the manufacture of electrospun sutures. The X-ray photoelectron spectroscopy (XPS) data collection was performed at the EPSRC National Facility for XPS ("HarwellXPS"), operated by Cardiff University and UCL, under Contract No. PR16195. AJC receives grant funding from the NIHR, the Wellcome Trust, the MRC/UKRI, and Versus Arthritis.

\section{CONFLICT OF INTEREST}

The authors declare no competing interests.

\section{ORCID}

Roxanna E. Abhari (D) https://orcid. org/0000-0002-4520-6534

\section{REFERENCES}

1. Yamaguchi K, Ditsios K, Middleton WD, Hildebolt CF, Galatz LM, Teefey SAl. The demographic and morphological features of rotator cuff disease. A comparison of asymptomatic and symptomatic shoulders. J Bone Joint Surg Am. 2006;88:1699-1704.

2. Ho JO, Sawadkar P, Mudera V. A review on the use of cell therapy in the treatment of tendon disease and injuries. J Tissue Eng. 2014;5:204173141454967

3. Carr AJ, Cooper CD, Campbell MK, et al. Clinical effectiveness and cost-effectiveness of open and arthroscopic rotator cuff repair [the UK Rotator Cuff Surgery (UKUFF) randomised trial]. Health Technol Assess. 2015;19:1-218.

4. Hopkins C, Fu S-C, Chua E, et al. Critical review on the socioeconomic impact of tendinopathy. Asia-Pacific J Sports Med Arthrosc Rehabil Technol. 2016;4:9-20.

5. Hollister SJ. Porous scaffold design for tissue engineering. Nat Mater. 2005;4:518-524.

6. Wong JKF, Cerovac S, Ferguson MWJ, McGrouther DA. The cellular effect of a single interrupted suture on tendon. J Hand Surg. 2006;31:358-367.

7. Rashid M, Dudhia J, Dakin SG, et al. Histological evaluation of cellular response to a multifilament electrospun suture for tendon repair. PLoS One. 2020;15:e234982.

8. Lock AM, Gao R, Naot D, Coleman B, Cornish J, Musson DS. Induction of immune gene expression and inflammatory mediator release by commonly used surgical suture materials: an experimental in vitro study. Patient Saf Surg. 2017;11.

9. Rothrauff BB, Lauro BB, Yang G, Debski RE, Musahl V, Tuan RS. Braided and Stacked Electrospun Nanofibrous Scaffolds for Tendon and Ligament Tissue Engineering. Tissue Eng Part A. 2017;23:378-389.

10. Wu S, Wang Y, Streubel PN, Duan B. Living nanofiber yarnbased woven biotextiles for tendon tissue engineering using cell tri-culture and mechanical stimulation. Acta Biomater. 2017;62:102-115.

11. Kendal A, Snelling S, Dakin S, Stace E, Mouthuy P-A, Carr A. Resorbable electrospun polydioxanone fibres modify the behaviour of cells from both healthy and diseased human tendons. Eur Cells Mater. 2017;33:169-182.

12. Beldjilali-Labro M, Garcia Garcia A, Farhat F, et al. Biomaterials in Tendon and Skeletal Muscle Tissue Engineering: Current Trends and Challenges. Materials (Basel). 2018;11:1116.

13. Mouthuy P-A, Zargar N, Hakimi O, Lostis E, Carr A. Fabrication of continuous electrospun filaments with potential for use as medical fibres. Biofabrication. 2015;7:25006.

14. Bosworth L, Alam N, Wong J, Downes S. Investigation of 2D and 3D electrospun scaffolds intended for tendon repair. J Mater Sci - Mater Med. 2013;24:1605-1614.

15. Shang S, Yang F, Cheng X, Walboomers X, Jansen J. The effect of electrospun fibre alignment on the behaviour of rat periodontal ligament cells. Eur Cells Mater. 2010;19:180-192.

16. Orr SB, Chainani A, Hippensteel KJ, et al. Aligned multilayered electrospun scaffolds for rotator cuff tendon tissue engineering. Acta Biomater. 2015;24:117-126. 
17. Hakimi O, Mouthuy PA, Zargar N, Lostis E, Morrey M, Carr A. A layered electrospun and woven surgical scaffold to enhance endogenous tendon repair. Acta Biomater. 2015;26:124-135.

18. Smith RD, Carr A, Dakin S, Snelling S, Yapp C, Hakimi O. The response of tenocytes to commercial scaffolds used for rotator cuff repair. Eur Cells Mater. 2016;31:107-118.

19. Abhari RE, Mouthuy P-A, Zargar N, Brown C, Carr A. Effect of annealing on the mechanical properties and the degradation of electrospun polydioxanone filaments. J Mech Behav Biomed Mater. 2017;67:127-134.

20. Smith R. Investigating the role of scaffolds for rotator cuff repair. Oxford: University of Oxford; 2016.

21. Ewels P, Magnusson M, Lundin S, Käller M. MultiQC: summarize analysis results for multiple tools and samples in a single report. Bioinformatics. 2016;32:3047-3048.

22. Love MI, Huber W, Anders S. Moderated estimation of fold change and dispersion for RNA-seq data with DESeq2. Genome Biol. 2014; 15:550.

23. Yu G, Wang LG, Han Y, He QY. clusterProfiler: an R package for comparing biological themes among gene clusters. Omics. 2012;16:284-287.

24. Amani H, Arzaghi H, Bayandori M, et al. Controlling Cell Behavior through the Design of Biomaterial Surfaces: A Focus on Surface Modification Techniques. Adv Mate Interfaces. 2019;6:1900572.

25. Chen M, Patra PK, Warner SB, Bhowmick S. Role of fiber diameter in adhesion and proliferation of NIH 3 T3 fibroblast on electrospun polycaprolactone scaffolds. Tissue Eng. 2007;13:579-587.

26. Erisken C, Zhang X, Moffat KL, Levine WN, Lu HH. Scaffold Fiber Diameter Regulates Human Tendon Fibroblast Growth and Differentiation. Tissue Eng Part A. 2013;19:519-528.

27. Dakin SG, Dudhia J, Smith RKW. Resolving an inflammatory concept: The importance of inflammation and resolution in tendinopathy. Vet Immunol Immunopathol. 2014;158:121-127.

28. Lim J, Munivez E, Jiang M-M, et al. mTORC1 Signaling is a Critical Regulator of Postnatal Tendon Development. Sci Rep. 2017;7:1-12.

29. Sanz LE, Patterson JA, Kamath R, Willett G, Ahmed SW, Butterfield AB. Comparison of Maxon suture with Vicryl, chromic catgut, and PDS sutures in fascial closure in rats. Obstet Gynecol. 1988;71:418-422.

30. van Steensel S, Hil L1, Bloemen A, et al. Prevention of incisional hernia using different suture materials for closing the abdominal wall: a comparison of PDS, Vicryl and Prolene in a rat model. Hernia. 2020;24:67-78.
31. O'Broin ES, Earley MJ, Smyth H, Hooper ACB. Absorbable sutures in tendon repair: A comparison of PDS with prolene in rabbit tendon repair. J Hand Surg. 1995;20:505-508.

32. Stone RC, Pastar I, Ojeh N, et al. Epithelial-Mesenchymal Transition in Tissue Repair and Fibrosis. Cell Tissue Res. 2016;365:495-506.

33. Lädermann A, Denard PJ, Collin P. Massive rotator cuff tears: definition and treatment. Int Orthop. 2015;39:2403-2414.

34. Stolk M, Klatte-Schulz F, Schmock A, Minkwitz S, Wildemann B, Seifert M. New insights into tenocyte-immune cell interplay in an in vitro model of inflammation. Sci Rep. 2017;7:1-14.

35. Oliva N, Carcole M, Beckerman M, et al. Regulation of dendrimer/ dextran material performance by altered tissue microenvironment in inflammation and neoplasia. Sci Transl Med. 2015;7:272ra211.

36. Ratner BD. Healing with medical implants: The body battles back. Sci Transl Med. 2015;7:272fs274.

37. Kendal AR, Layton T, Al-Mossawi H, Brown R. Identification of human tendon cell populations in healthy and diseased tissue using combined single cell transcriptomics and proteomics. bioRxiv. 2009;2019(2012):869933.

38. Swanson JB, De Micheli AJ, Disser NP, et al. A single-cell transcriptional atlas identifies extensive heterogeneity in the cellular composition of tendons. bioRxiv. 2019.

39. Yang G, Rothrauff BB, Tuan RS. Tendon and Ligament Regeneration and Repair: Clinical Relevance and Developmental Paradigm. Birth Defects Res Part C Embryo Today. 2013;99:203-222.

40. Lach AA, Morris HL, Martins JA, Stace ET, Carr AJ, Mouthuy PA. Pyridine as an additive to improve the deposition of continuous electrospun filaments. PLoS One. 2019;14:e0214419.

\section{SUPPORTING INFORMATION}

Additional supporting information may be found online in the Supporting Information section.

How to cite this article: Nezhentsev A, Abhari RE, Baldwin MJ, et al. In vitro evaluation of the response of human tendon-derived stromal cells to a novel electrospun suture for tendon repair. Transl Sports Med. 2021;4:409-418. https://doi.org/10.1002/tsm2.233 